\section{Subcutaneous emphysema of the chest: could it be pulmonary mucormycosis?}

A 54-year-old diabetic man presented with acute respiratory distress, progressive swelling of the chest wall, productive cough and low-grade fever.

Chest radiographs showed increasing subcutaneous emphysema and pneumomediastinum (figure 1). CT scanning revealed a thin-walled cavity in the right lung, communicating with the posterior segmental bronchus of the right upper lobe (figure 2a) with rupture into the chest wall (figure 2b). Bronchoscopy

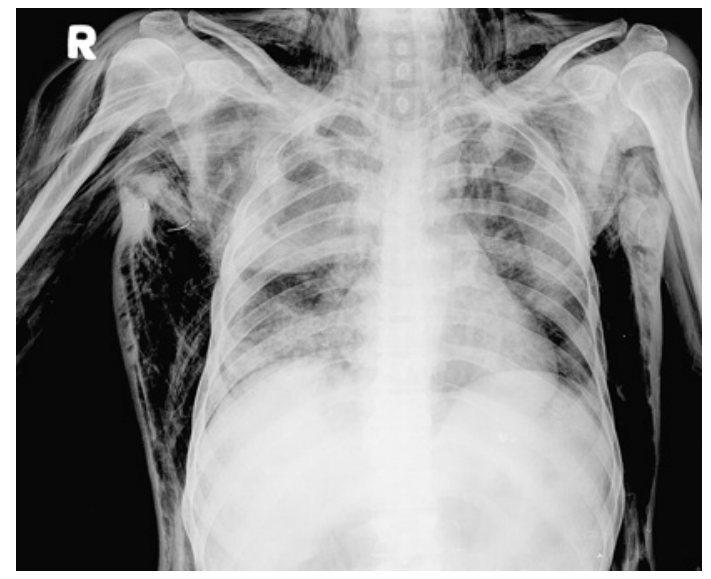

Figure 1 Chest radiograph showing subcutaneous emphysema which had started in the right chest wall and spread to the left side in a few hours.

\section{Learning points}

- Patients with diabetes, even if on treatment, are predisposed to mucormycosis irrespective of the presence of ketoacidosis. ${ }^{1}$

- Invasive pulmonary mucormycosis can sometimes present as progressive subcutaneous emphysema of the chest wall.

showed white-coloured membranes within the posterior segmental bronchus which were biopsied. Histopathology and fungal culture confirmed it to be invasive mucormycosis (figure 3a, b). Despite antifungal treatment, the patient died.

Progressive subcutaneous emphysema in pulmonary mucormycosis has not been reported in the literature and should be considered, especially if the patient is diabetic or immunocompromised. ${ }^{1} 2$ Early diagnosis and treatment will contribute towards a successful outcome.

\section{Chiramel George Koshy, Sanjeev Shah, Thomas Mammen}

Christian Medical College, Vellore 632 004, Tamil Nadu, India

Correspondence to Chiramel George Koshy, Department of Radiology, Christian Medical College, Vellore 632 004, Tamil Nadu, India; gkchiramel@gmail.com

Competing interests None.

Patient consent Obtained.

Provenance and peer review Not commissioned; externally peer reviewed.

Accepted 9 April 2009

Thorax 2010;65:280. doi:10.1136/thx.2009.115659

\section{REFERENCES}

1. Tedder M, Spratt JA, Anstadt MP, et al. Pulmonary mucormycosis: results of medical and surgical therapy. Ann Thorac Surg 1994;57:1044-50.

2. Lee FY, Mossad SB, Adal KA. Pulmonary mucormycosis: the last 30 years. Arch Intern Med 1999;159:1301-9.
Figure 2 CT scan of the chest showing a cavitary lesion in the upper lobe of the right lung communicating with the posterior segmental bronchus of the right upper lobe (arrow in figure 2a), with rupture into the chest wall (arrow in figure $2 \mathrm{~b}$ ).
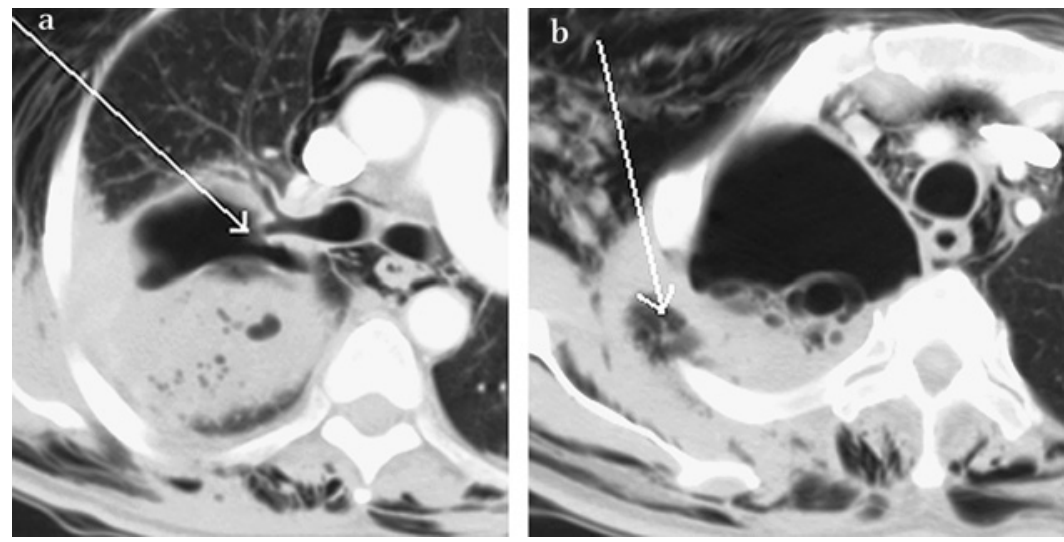

Figure 3 Histopathological evaluation of the biopsied membranes showing fungal hyphae suggestive of mucormycosis which are broader, less septate and branch less frequently and more unevenly than the hyphae of aspergillosis. (a) PAS stain, magnification $\times 400$; (b) GMS stain, magnification $\times 400$.
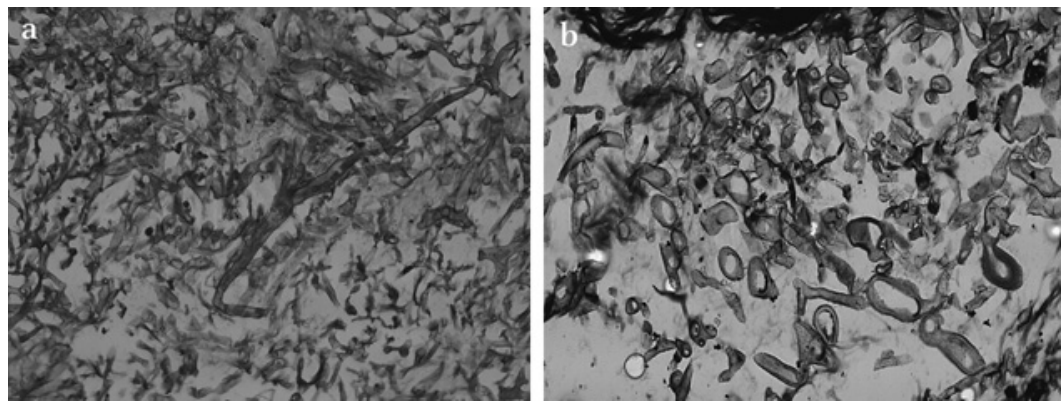